\title{
Canard Limit Cycle of the Holling-Tanner Model
}

\author{
Chongwu Zheng, ${ }^{1}$ Fengqin Zhang, ${ }^{1}$ and Jianquan $\mathrm{Li}^{2}$ \\ ${ }^{1}$ Department of Mathematics, Yuncheng University, Yuncheng, Shanxi 044000, China \\ ${ }^{2}$ Science College, Air Force Engineering University, Xian 710051, China \\ Correspondence should be addressed to Fengqin Zhang; zhafq@263.net
}

Received 11 April 2014; Accepted 2 July 2014; Published 16 July 2014

Academic Editor: Delfim F. M. Torres

Copyright (C) 2014 Chongwu Zheng et al. This is an open access article distributed under the Creative Commons Attribution License, which permits unrestricted use, distribution, and reproduction in any medium, provided the original work is properly cited.

By using the singular perturbation theory on canard cycles, we investigate the canard phenomenon for the Holling-Tanner model with the intrinsic growth rate of the predator small enough. The obtained result shows that there may be at most one canard limit cycle, and the range of small parameters is estimated. The phenomenon of outbreak is explained.

\section{Introduction}

The predator-prey system

$$
\begin{gathered}
\frac{d x}{d t}=r_{1} x\left(1-\frac{x}{K}\right)-\frac{c_{1} x y}{x+a_{1}}, \\
\frac{d x}{d t}=s_{1} y\left(1-\frac{y}{\gamma x}\right)
\end{gathered}
$$

is referred to as the Holling-Tanner model [1]. In (1), the variables $x(t)$ and $y(t)$ denote the numbers of preys and predators at time $t$, respectively. $r_{1}$ is the intrinsic growth rate of the prey, $K$ is the carrying capacity of the prey, and function $c_{1} x /\left(x+a_{1}\right)$, known as the functional response of Holling type II, is the rate at which predators consume the prey, where $c_{1}$ is the maximum number of a predator and $a_{1}$ is the half saturation value corresponding to the number of preys necessary to achieve one half of the maximum value. It is assumed that the predator grows logistically with intrinsic growth rate $s_{1}$ and carrying capacity $\gamma x$ taking on the role of a prey-dependent carrying capacity for the predator, where $\gamma$ is a measure of the quality of the prey as food for the predator. From the biological meaning all the parameters of (1) are positive.

It is easy to see that the set $\{(x, y): 0<x \leq K, y>0\}$ is positively invariant for (1), and there is always a unique positive equilibrium. The local stability of the positive equilibrium was analyzed in [2, 3]. In [4] Hsu and Huang considered the question of global stability of the equilibrium and obtained conditions under which its local stability implies the global stability. In 2003 Braza [5] investigated the existence of at least one limit cycle of (1) in a Hopf bifurcation. Gasull et al. [6] showed that (1) can exhibit two limit cycles by constructing an example. The bifurcation diagram of limit cycles of (1) was described to show the regions of parameters where two limit cycles or a semistable limit cycle appears [7]. Applying Andronov-Hopf bifurcation Theorem Hsu and Hwang proved that the Hopf bifurcation is subcritical and thus (1) may have at least two limit cycles for some parameter ranges and explained the phenomenon of outbreaking in [8] obtained by numerical simulations [9].

In the references mentioned above, it was assumed that all the parameters are positive constants, but there is not much consideration for the system with small parameters. It is interesting and important to analyze the bifurcation of limit periodic set of the system in the case when $s_{1}$ is very small; that is, the intrinsic growth rate of the predator remains at a very low level. For this case when plotting the trajectories of system (1) with the intrinsic growth rate small enough by numerical simulations, it would take much longer time when the trajectory passes by the vertical $y$-axis. This behaves like the usual relaxation oscillation phenomena observed in slowfast systems. By using the singular perturbation theory [10] Li and Zhu [11] studied the canard limit cycles for predator-prey system with functional response of four Holling types and developed criteria for the existence and multiplicity of canard 
slow-fast cycle (i.e., limit periodic set) in general predatorprey systems.

Although some references have considered the existence of two limit cycles for $(1)[6,7,9]$, it is still interesting to discuss the existence of canard slow-fast cycle, since the canard slow-fast cycle consists of a part of the fast orbit and a part of the slow manifold and is the limit of a family of periodic orbits as the value of a positive parameter tends to zero. In this paper we mainly consider the existence of canard slow-fast cycle of (1) when $s_{1}$ is very small. Our result shows that there may be at most one canard limit cycle, and the range of small parameters is estimated. In next section we first reduce the number of parameters to three ones by some transformation for (1) and introduce some concepts of canard slow-fast cycle. In Section 3 we provide the complete inferences for the main result of this paper. Finally we give discussion according to the results of numerical simulation.

\section{Simplification of the Model and Canard Limit Cycle}

For the case where $s_{1}$ is very small, let

$$
s_{1}=\varepsilon_{1} s_{2}, \quad a_{1}=a_{2}-\varepsilon_{1} \lambda_{1},
$$

where $\varepsilon_{1}$ is positive and $\lambda_{1}$ may be negative, and we will choose $\varepsilon_{1}$ and $\left|\lambda_{1}\right|$ small enough to make $a_{2}>0$ in later discussion; then system (1) becomes

$$
\begin{gathered}
\frac{d x}{d t}=r_{1} x\left(1-\frac{x}{K}\right)-\frac{c_{1} x y}{x+a_{2}-\varepsilon_{1} \lambda_{1}}, \\
\frac{d x}{d t}=\varepsilon_{1} s_{2} y\left(1-\frac{y}{\gamma x}\right) .
\end{gathered}
$$

For system (3), making the scaling

$$
x=K \bar{x}, \quad y=\frac{r_{1} K}{c_{1}} \bar{y}, \quad t=\frac{1}{r_{1}} \bar{t},
$$

and then dropping their bars yield

$$
\begin{gathered}
\frac{d x}{d t}=x(1-x)-\frac{x y}{x+a-\varepsilon \lambda}, \\
\frac{d y}{d t}=\varepsilon y\left(\delta-\frac{y}{x}\right),
\end{gathered}
$$

where

$$
\varepsilon=\frac{\varepsilon_{1} s_{2}}{c_{1} \gamma}, \quad \lambda=\frac{c_{1} \gamma \lambda_{1}}{K s_{2}}, \quad a=\frac{a_{2}}{K}, \quad \delta=\frac{c_{1} \gamma}{r_{1}} .
$$

Since $x>0$ and $a>\varepsilon \lambda$, letting $d t=((x+a-\varepsilon \lambda) / x) d \tau$ gives the equivalent system of (5):

$$
\begin{aligned}
& \frac{d x}{d \tau}=f_{\varepsilon}(x)-y, \\
& \frac{d y}{d \tau}=\varepsilon g(x, y),
\end{aligned}
$$

where

$$
\begin{gathered}
f_{\varepsilon}(x)=(1-x)(x+a-\varepsilon \lambda), \\
g(x, y)=\frac{y}{x^{2}}(x+a-\varepsilon \lambda)(\delta x-y) .
\end{gathered}
$$

If we introduce slow time $t=\varepsilon \tau$, then system (7) is transformed to

$$
\begin{gathered}
\varepsilon \frac{d x}{d t}=f_{\varepsilon}(x)-y, \\
\frac{d y}{d t}=g(x, y) .
\end{gathered}
$$

By lettng $\varepsilon=0$ for (7) and (9), we obtain the layer equations

$$
\begin{gathered}
\frac{d x}{d \tau}=f_{0}(x)-y, \\
\frac{d y}{d \tau}=0,
\end{gathered}
$$

and the reduced equations

$$
\begin{aligned}
y & =f_{0}(x), \\
\frac{d y}{d t} & =g(x, y),
\end{aligned}
$$

respectively.

In this paper we will always assume that $a<1$. The slow manifold $S=\left\{(x, y): y=f_{0}(x)\right\}$ has a unique extreme point at

$$
\left(x_{0}, y_{0}\right)=\left(\frac{1-a}{2}, \frac{(1+a)^{2}}{4}\right),
$$

and it is a simple maximum point; see Figure 1(a). Besides, $S$ is globally concave for $x \geq 0$. The fast movement of the layer equations (10) is shown in Figure 1(b), where the orbits outside $S$ are parallel lines $\{y=c\}$ with different constant $c$, and the curve $S$ consists of singular points; hence it is also called the critical curve. Note that the points on $S$ left to $\left(x_{0}, y_{0}\right)$ are normally hyperbolic repelling while the points on $S$ right to $\left(x_{0}, y_{0}\right)$ are normally hyperbolic attracting.

To study the slow movement on $S \backslash\left\{\left(x_{0}, y_{0}\right)\right\}$, from the reduced equations (11) we have

$$
f_{0}^{\prime}(x) \frac{d x}{d t}=\left.\frac{d y}{d t}\right|_{y=f_{0}(x)}=g\left(x, f_{0}(x)\right),
$$

and hence along $S$ the slow movement is controlled by

$$
\frac{d x}{d t}=\frac{g\left(x, f_{0}(x)\right)}{f_{0}^{\prime}(x)} .
$$

In order to show the existence of canard slow-fast cycle, from Figure 1(b) it is necessary to illustrate that the right hand side of (14) should be negative near the point $\left(x_{0}, y_{0}\right)$.

In the next section, we will make some transformations such that the function $g(x, y)$ in system $(7)$ becomes $g(x, y)=$ 


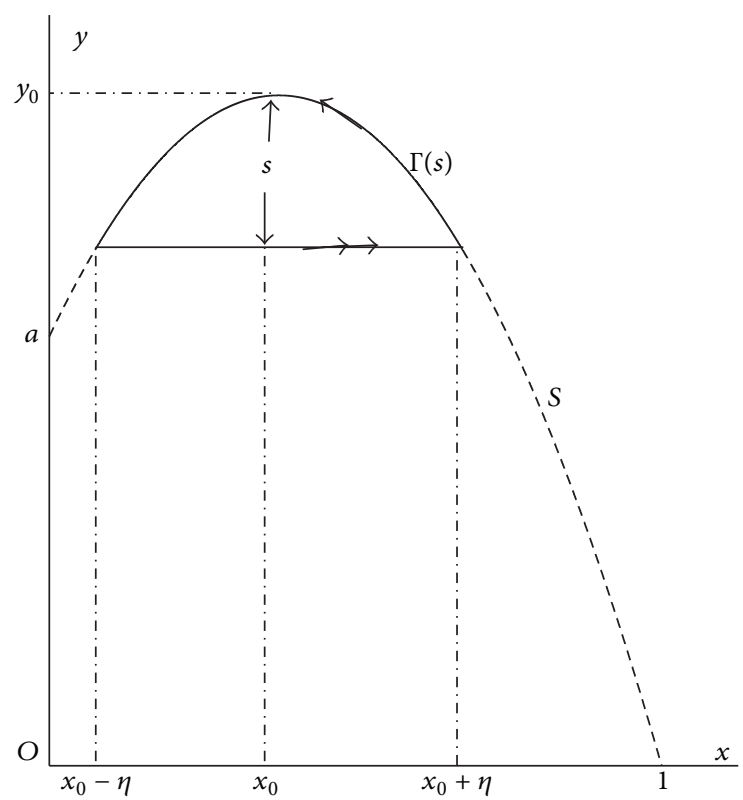

(a)

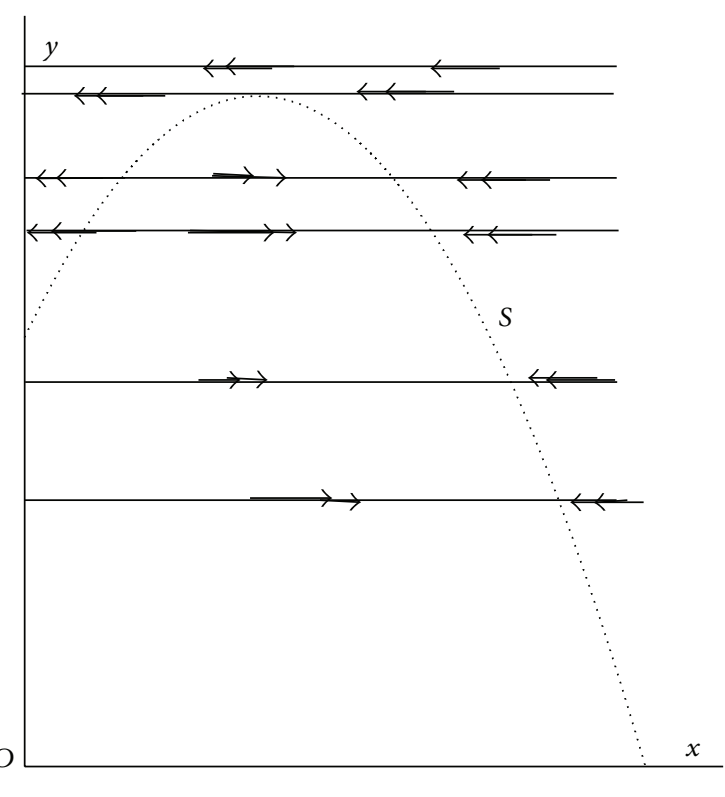

(b)

Figure 1: The limit periodic cycle $\Gamma(s)$ and the slow manifold $S$.

$\widetilde{g}(x, y, \lambda)$, and for $\lambda=0$ the right hand side of (14) is negative in an interval of $x$, containing $x_{0}$; then we may construct a slow-fast cycle (i.e., limit periodic set) $\Gamma(s)$ by combining a part of the fast orbit $y=y_{0}-s$, where $s \in\left(0, y_{0}-a\right)$, and a part of the slow manifold $S$, shown in Figure 1(a). As we mentioned above, on the left and right sides of the point $\left(x_{0}, y_{0}\right)$ the points on the slow manifold $S$ are normally hyperbolic repelling and attracting, respectively, the point $\left(x_{0}, y_{0}\right)$ is called a canard point, and the slow-fast cycle $\Gamma(s)$ is called a canard type, or simply called a canard slow-fast cycle; see $[10,12]$.

\section{Existence and Uniqueness of Canard Slow-Fast Cycles}

To study the local behavior of system (7) near $\left(x_{0}, y_{0}\right)$, we make the change of coordinates

$$
u=x-x_{0}, \quad v=y-y_{0} .
$$

For $(u, v)$ near $(0,0)$ system $(7)$ becomes

$$
\begin{gathered}
\frac{d u}{d \tau}=-v-u^{2}+\varepsilon \lambda\left(u-1+x_{0}\right), \\
\frac{d v}{d \tau}=\frac{\varepsilon}{x_{0}^{4}}\left[\bar{b}_{0}+\bar{b}_{1} u+\bar{b}_{2} u^{2}\right. \\
\left.+v\left(\bar{d}_{0}+\bar{d}_{1} u+\bar{d}_{2} v\right)+o\left(|u, v|^{2}\right)\right],
\end{gathered}
$$

where

$$
\begin{aligned}
& \bar{b}_{0}=b_{0}-\varepsilon \lambda\left(\delta x_{0}-y_{0}\right) x_{0}^{2} y_{0}, \\
& b_{0}=\left(x_{0}+a\right)\left(\delta x_{0}-y_{0}\right) x_{0}^{2} y_{0},
\end{aligned}
$$

$$
\begin{gathered}
\bar{b}_{1}=b_{1}+\varepsilon \lambda\left(\delta x_{0}-2 y_{0}\right) x_{0} y_{0}, \\
b_{1}=x_{0} y_{0}\left(x_{0} y_{0}-\delta a x_{0}+2 a y_{0}\right), \\
\bar{b}_{2}=b_{2}+\varepsilon \lambda y_{0}\left(3 y_{0}-\delta x_{0}\right), \\
b_{2}=y_{0}\left(\delta a x_{0}-x_{0} y_{0}-3 a y_{0}\right), \\
\bar{d}_{0}=d_{0}-\varepsilon \lambda x_{0}^{2}\left(\delta x_{0}-2 y_{0}\right), \\
d_{0}=\left(x_{0}+a\right) x_{0}^{2}\left(\delta x_{0}-2 y_{0}\right), \\
\bar{d}_{1}=d_{1}+\varepsilon \lambda x_{0}\left(\delta x_{0}-4 y_{0}\right), \\
d_{1}=x_{0}\left(2 x_{0} y_{0}+4 a y_{0}-\delta a x_{0}\right), \\
\bar{d}_{2}=d_{2}+\varepsilon \lambda x_{0}^{2}, \quad d_{2}=-\left(x_{0}+a\right) x_{0}^{2} .
\end{gathered}
$$

Notice that $b_{1}=x_{0} y_{0}^{2}\left(x_{0}+a\right)>0$ as $b_{0}=0$ (i.e., $\delta x_{0}=y_{0}$ ); then $b>0$ for some $\delta$ and $a$ making $\left|b_{0}\right|$ small enough. For such $\delta$ and $a$, making one more change of coordinates

$$
u=-\frac{\sqrt{b_{1}}}{x_{0}^{2}} x, \quad v=-\frac{b_{1}}{x_{0}^{4}} y, \quad \tau=\frac{x_{0}^{2}}{\sqrt{b_{1}}} t
$$

equation (16) becomes

$$
\frac{d x}{d t}=-y+x^{2}+\varepsilon \lambda \frac{x_{0}^{4}}{b_{1}}\left(\frac{\sqrt{b_{1}}}{x_{0}^{2}} x+1-x_{0}\right),
$$




$$
\begin{gathered}
\frac{d y}{d t}=\varepsilon\left[\frac{\bar{b}_{1}}{b_{1}} x-\frac{\bar{b}_{0} x_{0}^{2}}{b_{1} \sqrt{b_{1}}}-\frac{\bar{b}_{2}}{\sqrt{b_{1}} x_{0}^{2}} x^{2}\right. \\
+\frac{y}{\sqrt{b_{1}} x_{0}^{2}}\left(\bar{d}_{0}-\frac{\bar{d}_{1} \sqrt{b_{1}}}{x_{0}^{2}} x-\frac{\bar{d}_{2} b_{1}}{x_{0}^{4}} y\right) \\
\left.+o\left(|x, y|^{2}\right)\right] .
\end{gathered}
$$

In addition, we choose $\delta$ and $a$ such that

$$
\frac{b_{0} x_{0}^{2}}{b_{1} \sqrt{b_{1}}}=\lambda
$$

and then (19) can be rewritten as

$$
\begin{aligned}
\frac{d x}{d t}= & -y h_{1}(x, y, \lambda, \varepsilon)+x^{2} h_{2}(x, y, \lambda, \varepsilon) \\
& +\varepsilon h_{3}(x, y, \lambda, \varepsilon), \\
\frac{d y}{d t}= & \varepsilon\left[x h_{4}(x, y, \lambda, \varepsilon)-\lambda h_{5}(x, y, \lambda, \varepsilon)\right. \\
& \left.+y h_{6}(x, y, \lambda, \varepsilon)+o\left(|x, y|^{2}\right)\right],
\end{aligned}
$$

where

$$
\begin{aligned}
h_{1}(x, y, \lambda, \varepsilon) & =1 \\
h_{2}(x, y, \lambda, \varepsilon) & =1 \\
h_{3}(x, y, \lambda, \varepsilon) & =\lambda \frac{x_{0}^{4}}{b_{1}}\left(\frac{\sqrt{b_{1}}}{x_{0}^{2}} x+1-x_{0}\right) \\
h_{4}(x, y, \lambda, \varepsilon) & =1+\frac{\bar{b}_{1}-b_{1}}{b_{1}}-\frac{\bar{b}_{2}}{\sqrt{b_{1}} x_{0}^{2}} x \\
& =1+\frac{\varepsilon \lambda\left(\delta x_{0}-2 y_{0}\right) x_{0}}{b_{1}}-\frac{\bar{b}_{2}}{\sqrt{b_{1}} x_{0}^{2}} x, \\
h_{5}(x, y, \lambda, \varepsilon) & =1+\frac{\left(\bar{b}_{0}-b_{0}\right) x_{0}^{2}}{b_{1} \sqrt{b_{1}} \lambda}=1-\frac{\varepsilon\left(\delta x_{0}-y_{0}\right) x_{0}^{4} y_{0}}{b_{1} \sqrt{b_{1}}}, \\
h_{6}(x, y, \lambda, \varepsilon) & =\frac{1}{\sqrt{b_{1}} x_{0}^{2}}\left(\bar{d}_{0}-\frac{\bar{d}_{1} \sqrt{b_{1}}}{x_{0}^{2}} x-\frac{\bar{d}_{2} b_{1}}{x_{0}^{4}} y\right) .
\end{aligned}
$$

From (17) and (20) we have

$$
\begin{aligned}
\lambda=0 \Longleftrightarrow b_{0}= & 0 \Longleftrightarrow \delta x_{0}=y_{0} \Longleftrightarrow \delta=\frac{(a+1)^{2}}{2(1-a)}=: \delta_{0} \\
& \left.b_{1}\right|_{\lambda=0}=x_{0} y_{0}^{2}\left(x_{0}+a\right)>0 \\
& \left.b_{2}\right|_{\lambda=0}=-y_{0}^{2}\left(x_{0}+2 a\right) \\
& \left.d_{0}\right|_{\lambda=0}=-\left(x_{0}+a\right) x_{0}^{2} y_{0} .
\end{aligned}
$$

Furthermore, $\left.b_{1}\right|_{\lambda=0}>0$ implies that the change of coordinates (18) is available.

According to (3.12) and (3.13) of [10], from (24) we have

$$
\begin{aligned}
& a_{1}=\frac{\partial h_{3}}{\partial x}(0,0,0,0)=0, \\
& a_{2}=\frac{\partial h_{1}}{\partial x}(0,0,0,0)=0, \\
& a_{3}=\frac{\partial h_{2}}{\partial x}(0,0,0,0)=0, \\
& a_{4}=\frac{\partial h_{4}}{\partial x}(0,0,0,0)=\frac{(1+a)(1+3 a) \sqrt{1-a^{2}}}{(1-a)^{3}}, \\
& a_{5}=h_{6}(0,0,0,0)=-\frac{\sqrt{1-a^{2}}}{1-a},
\end{aligned}
$$

and then

$$
A=-a_{2}+3 a_{3}-2 a_{4}-2 a_{5}=-\frac{4 a(a+3) \sqrt{1-a^{2}}}{(1-a)^{3}}<0 .
$$

Therefore, the canard cycle of (7) is asymptotically stable if it exists.

On the other hand, for (14) we have

$$
\begin{aligned}
\left.\frac{d x}{d t}\right|_{\lambda=0} & =\left.\frac{g\left(x, f_{0}(x)\right)}{f_{0}^{\prime}(x)}\right|_{\lambda=0} \\
& =-\frac{(1-x)(a+x)^{2}[2 a+(1-a) x]}{2(1-a) x^{2}}<0
\end{aligned}
$$

for $a<1$ and $x<1$. The fact that $f_{0}^{\prime}(x) \neq 0$ for $x>0$ and $x \neq x_{0}$ implies that system (14) along the slow manifold $S$ is well defined near $x_{0}$ and that the point $\left(x_{0}, y_{0}\right)$ is a canard one.

Further, by Theorems 3.3 of [10], $A<0$ implies that a $\mathcal{E}$ family of canard cycles bifurcates from the limit periodic set $\Gamma(s)$, for each $s \in\left(0, y_{0}-a\right)$, and for some small $\lambda=\lambda(s, \sqrt{\varepsilon})$ and $0<\varepsilon \ll 1$. Moreover, $\lambda(s, \sqrt{\varepsilon})$ satisfies

$$
\left|\lambda(s, \sqrt{\varepsilon})-\lambda_{c}(\sqrt{\varepsilon})\right| \leq e^{-1 / \varepsilon^{\sigma}}
$$

where $\sigma \in(0,1)$ is a constant and

$$
\begin{aligned}
\lambda_{c}(\sqrt{\varepsilon}) & =-\left(\frac{a_{1}+a_{5}}{2}+\frac{A}{8}\right) \varepsilon+O\left(\varepsilon^{3 / 2}\right) \\
& =\frac{\left(2 a^{2}+a+1\right) \sqrt{1-a^{2}}}{2(1-a)^{3}} \varepsilon+O\left(\varepsilon^{3 / 2}\right) .
\end{aligned}
$$

The inequality (28) implies that, when there is a family of canard cycles bifurcating from the canard slow-fast cycle, $\lambda$ in (7) depends on $\varepsilon$, and $\lambda \rightarrow 0$ as $\varepsilon \rightarrow 0$. 


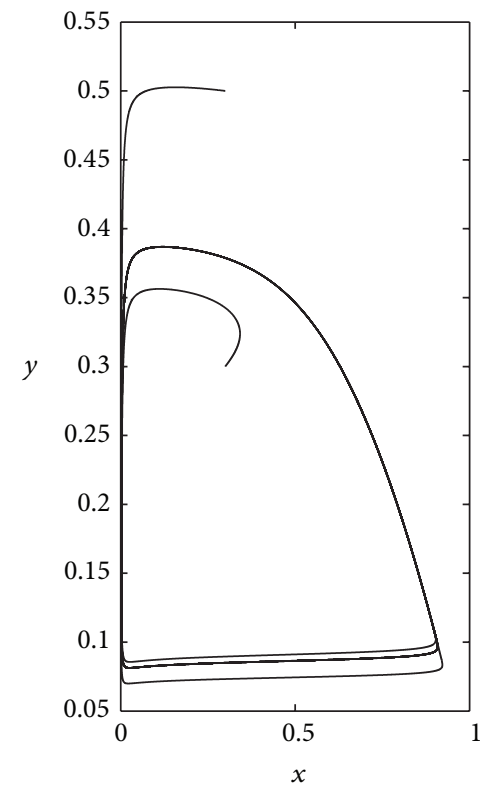

(a)

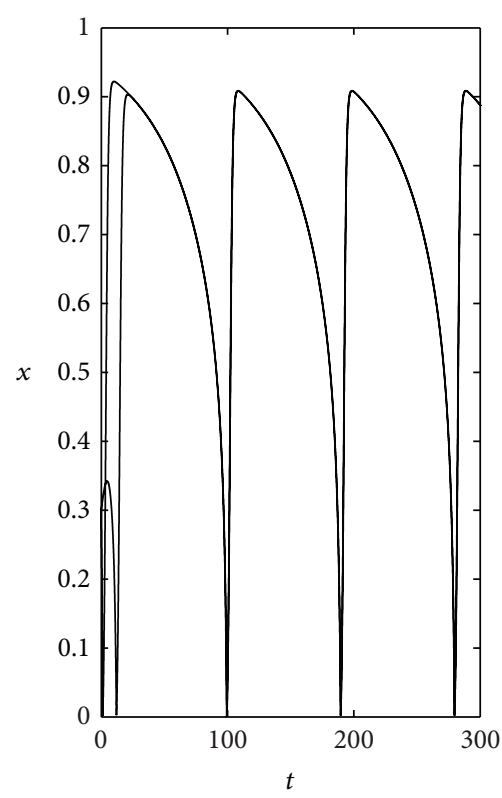

(b)

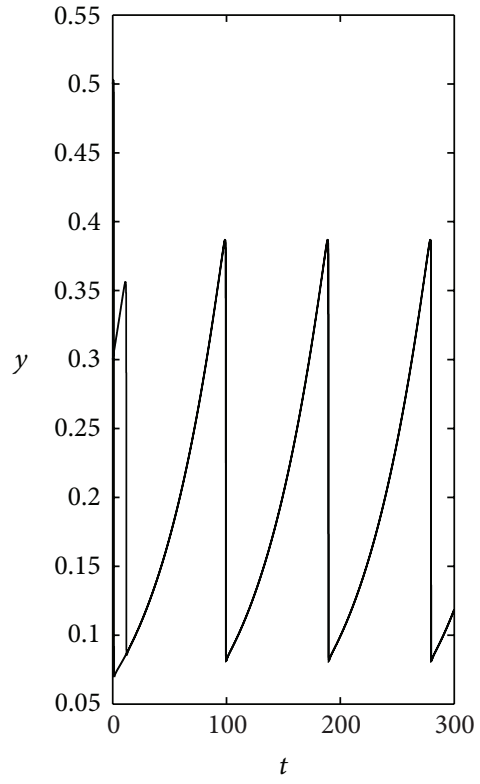

(c)

FIGURE 2: The trajectories of (5) with the fast stage and the slow stage and the corresponding curves of $x=x(t)$ and $y=y(t)$, where $\delta=3.2$, $a=0.15, \epsilon=0.005$, and $\lambda=0.001$.

Further, direct computation shows

$$
\begin{aligned}
& \left.\frac{\left[f_{0}^{\prime}(x)\right]^{2}}{g\left(x, f_{0}(x)\right)}\right|_{\lambda=0} \\
& =\frac{2(1-a)[2 x-(1-a)] x^{2}}{(1-x)(x+a)^{2}[(1-a) x+2 a]} \\
& =2(1-a)\left[\frac{1}{(a+1)^{2}(1-x)}+\frac{2 a+3}{(a+1)^{2}(x+a)}\right. \\
& \quad-\frac{a}{(a+1)(x+a)^{2}} \\
& \left.\quad-\frac{4}{(a+1)[(1-a) x+2 a]}\right] .
\end{aligned}
$$

According to the symmetry of the slow manifold $S$ about $x=$ $(1-a) / 2$, for $\lambda=0$ (i.e., $\left.\delta=\delta_{0}\right)$ we suppose that the interval of $x$-coordinate of the slow-fast cycle $\Gamma(s)$ is $[(1-a) / 2-\eta,(1-$ a) $/ 2+\eta]$, where $\eta \in(0,(1-a) / 2)$ and $s \in\left(0, y_{0}-a\right)$; see Figure 1(a). By straightforwardly calculating, we have

$$
I\left(s, \delta_{0}\right)=\left.\int_{(1-a) / 2+\eta}^{(1-a) / 2-\eta} \frac{\left(f_{0}^{\prime}(x)\right)^{2}}{g\left(x, f_{0}(x)\right)}\right|_{\lambda=0} d x=2(a-1) h(\eta)
$$

where

$$
\begin{aligned}
h(\eta)= & \frac{2(a+2)}{(a+1)^{2}} \ln \frac{1+a+2 \eta}{1+a-2 \eta} \\
& -\frac{4}{1-a^{2}} \ln \frac{(a+1)^{2}+2 \eta(1-a)}{(a+1)^{2}-2 \eta(1-a)} \\
& -\frac{8 \eta a}{(a+1)\left[(1+a)^{2}-4 \eta^{2}\right]} .
\end{aligned}
$$

Note that

$$
h^{\prime}(\eta)=-\frac{64 a \eta^{2}\left[4(3-a) \eta^{2}-\left(1-a^{2}\right)(3+a)\right]}{\left[(1+a)^{2}-4 \eta^{2}\right]^{2}\left[(a+1)^{4}-4 \eta^{2}(1-a)^{2}\right]}>0
$$

for $0<a<1$ and $0<\eta<(1-a) / 2$; then $h(0)=0$ implies that $h(\eta)>0$ for $0<\eta<(1-a) / 2$; that is, $I\left(s, \delta_{0}\right)<0$ for $0<s<y_{0}-a$. Hence, it follows form the results in [12] that (7) has at most one canard slow-fast cycle.

Summarizing the above inference, we obtain the following result.

Theorem 1. When $a<1$, (7) has at most one canard slowfast cycle, which is asymptotically stable for $0<\varepsilon \ll 1$ and $\lambda$ satisfying (28).

\section{Numerical Simulation and Discussion}

We first plot the trajectories of (5) and the corresponding curves of $x=x(t)$ and $y=y(t)$ as $\delta=3.2, a=0.15$, 


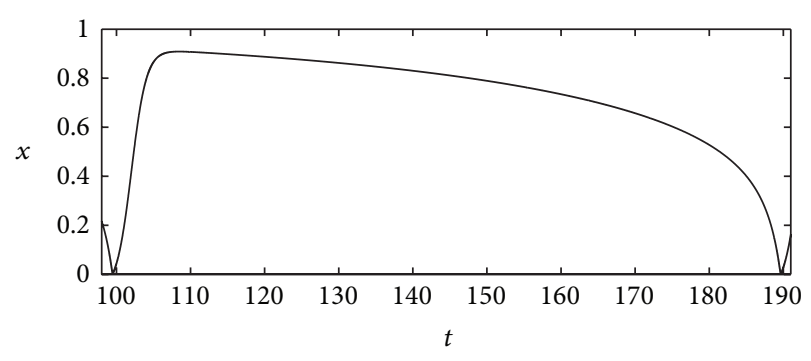

(a)

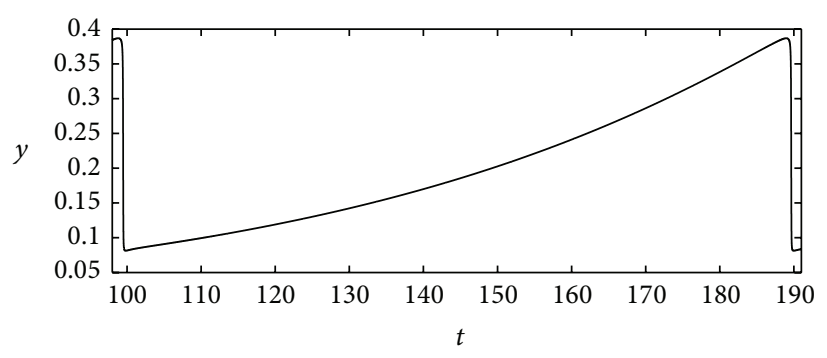

(b)

FIgURE 3: The curves of $x=x(t)$ and $y=y(t)$ obtained by locally enlarging the curves of $x=x(t)$ and $y=y(t)$ in Figure 2, respectively.

$\epsilon=0.005$, and $\lambda=0.001$ (see Figure 2), in which Figures 2(b) and $2(\mathrm{c})$ illustrate the existence of the fast stages of $x=x(t)$ and $y=y(t)$.

Note that the vertical and horizontal isoclines of (5) divide the first quadrant of the $(x, y)$-plane into four regions:

$$
\begin{aligned}
& D_{1}=\left\{(x, y) \in R_{+}^{2}: \frac{d x}{d t}>0, \frac{d y}{d t}>0\right\} ; \\
& D_{2}=\left\{(x, y) \in R_{+}^{2}: \frac{d x}{d t}<0, \frac{d y}{d t}>0\right\} ; \\
& D_{3}=\left\{(x, y) \in R_{+}^{2}: \frac{d x}{d t}<0, \frac{d y}{d t}<0\right\} ; \\
& D_{4}=\left\{(x, y) \in R_{+}^{2}: \frac{d x}{d t}>0, \frac{d y}{d t}<0\right\} .
\end{aligned}
$$

And from (2) and (6) it follows that $r_{1}=c_{1} \gamma / \delta, a_{1}=(a-\varepsilon \lambda) K$, and $s_{1}=c_{1} \gamma \varepsilon$, where $\alpha, \delta, \varepsilon$, and $\lambda$ are the parameters in (5); then, corresponding to (1), the value of parameter $s_{1}$ is very small relative to other parameters as $\varepsilon$ is very small.

In order to more clearly show the fast stages, we locally enlarge the two curves in Figures 2(b) and 2(c) into Figures 3 (a) and 3(b), respectively. From Figures 3(a) and 3(b), we can know that the fast stages of $x=x(t)$ occur in region $D_{1}$, and those of $y=y(t)$ occur in region $D_{3}$. Biologically, $x=x(t)$ and $y=y(t)$ represent the numbers of preys and predators at time $t$, respectively; then, when the intrinsic growth rate of the predator is very small and there exists the periodic solution for (1), according to signs of derivatives of $x(t)$ and $y(t)$ with respect to time $t$, the number of the preys increases quickly from the number small enough as $(x(t), y(t)) \epsilon$ $D_{1}$, and the number of the predators decreases quickly as $(x(t), y(t)) \in D_{3}$. The quick increase of the prey from the small value may explain the phenomenon of outbreak in [8].

\section{Conflict of Interests}

The authors declare that there is no conflict of interests regarding the publication of this paper.

\section{Acknowledgment}

This work is supported by the National Natural Science Fund of China (nos. 11371369 and 11371313).

\section{References}

[1] J. T. Tanner, "The stability and the intrinsic growth rates of prey and predator populations," Ecology, vol. 5, pp. 855-867, 1975.

[2] J. D. Murray, Mathematical Biology, Springer, Berlin, Germany, 1989.

[3] R. M. May, Stability and Complexity in Model Ecosystems, Princeton University Press, Princeton, NJ, USA, 1974.

[4] S. B. Hsu and T. W. Huang, "Global stability for a class of predator-prey systems," SIAM Journal on Applied Mathematics, vol. 55, no. 3, pp. 763-783, 1995.

[5] P. A. Braza, "The bifurcation structure of the Holling-Tanner model for predator-prey interactions using two-timing," SIAM Journal on Applied Mathematics, vol. 63, no. 3, pp. 889-904, 2003.

[6] A. Gasull, R. E. Kooij, and J. Torregrosa, "Limit cycles in the Holling-Tanner model," Publicacions Matemàtiques, vol. 41, pp. 149-167, 1997.

[7] E. Sáez and E. González, "Dynamics of a predator-prey model," SIAM Journal on Applied Mathematics, vol. 59, no. 5, pp. 18671878, 1999.

[8] D. J. Wollkind, J. B. Collings, and J. A. Logan, "Metastability in a temperature-dependent model system for predator-prey mite outbreak interactions on fruit trees," Bulletin of Mathematical Biology, vol. 50, no. 4, pp. 379-409, 1988.

[9] S. Hsu and T. Hwang, "Hopf bifurcation analysis for a predatorprey system of Holling and Leslie type," Taiwanese Journal of Mathematics, vol. 3, no. 1, pp. 35-53, 1999.

[10] M. Krupa and P. Szmolyan, "Relaxation oscillation and canard explosion," Journal of Differential Equations, vol. 174, no. 2, pp. 312-368, 2001.

[11] C. Li and H. Zhu, "Canard cycles for predator-prey systems with Holling types of functional response," Journal of Differential Equations, vol. 254, no. 2, pp. 879-910, 2013.

[12] P. De Maesschalck and F. Dumortier, "Canard cycles in the presence of slow dynamics with singularities," Proceedings of the Royal Society of Edinburgh A. Mathematics, vol. 138, no. 2, pp. 265-299, 2008. 


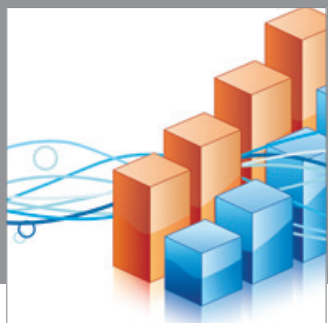

Advances in

Operations Research

mansans

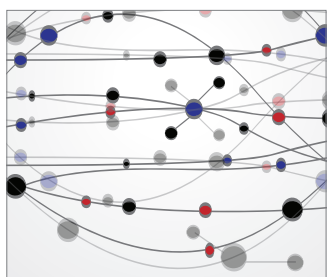

The Scientific World Journal
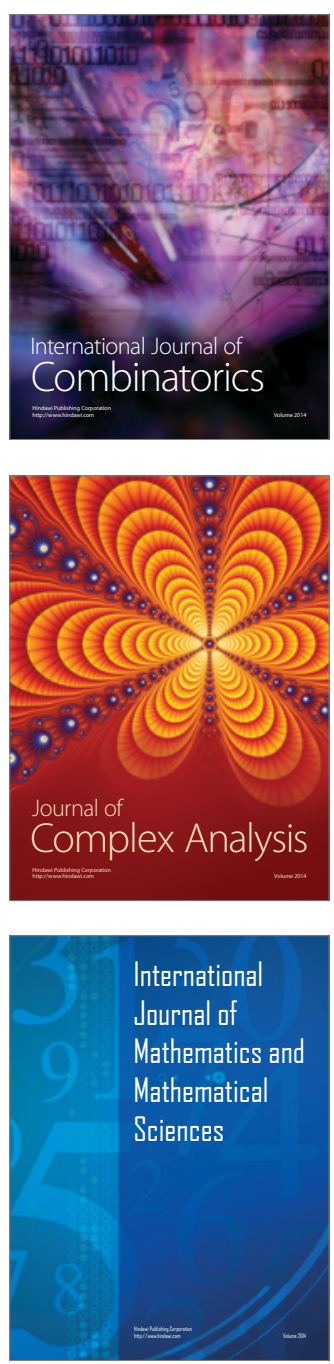
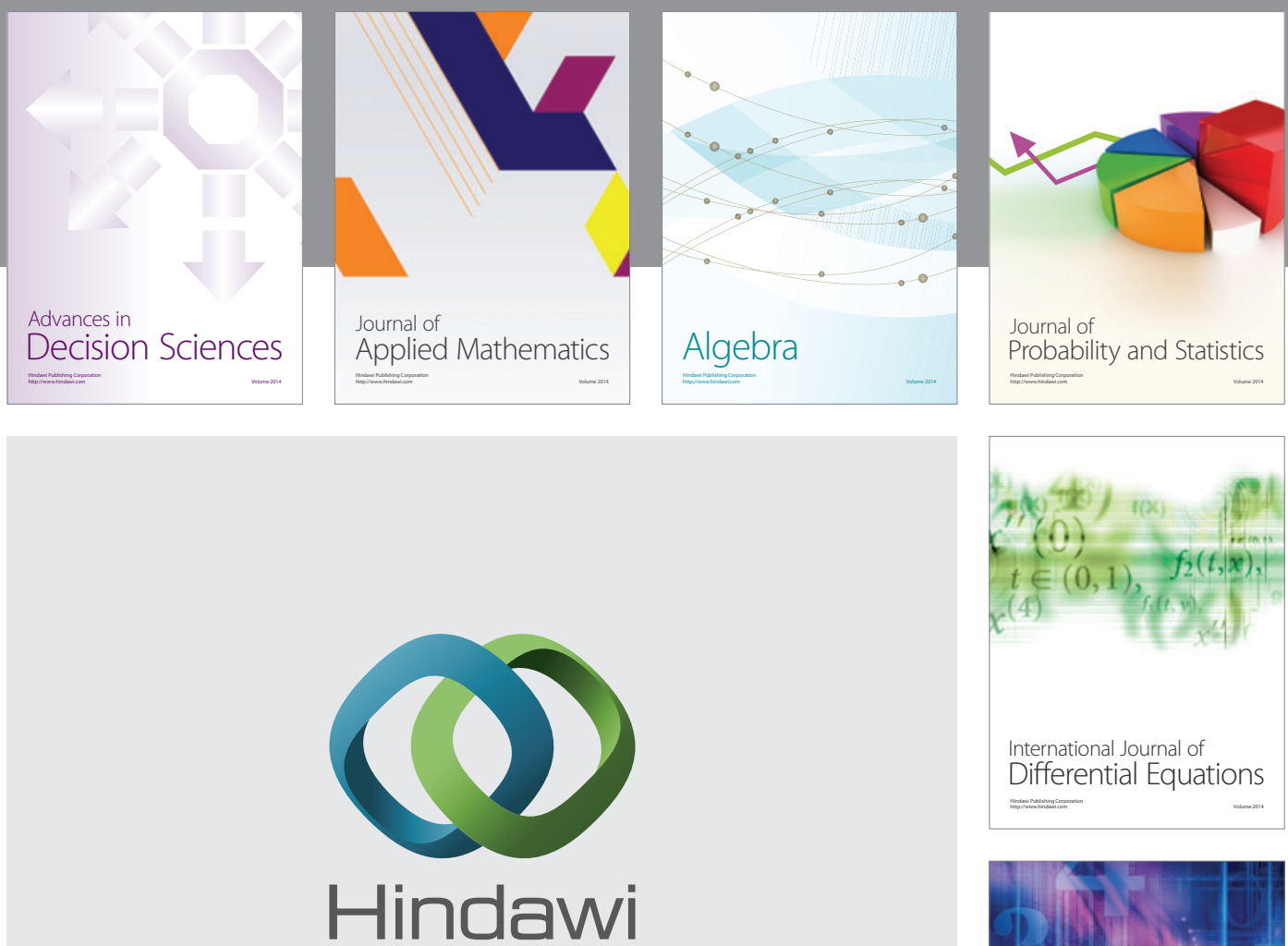

Submit your manuscripts at http://www.hindawi.com
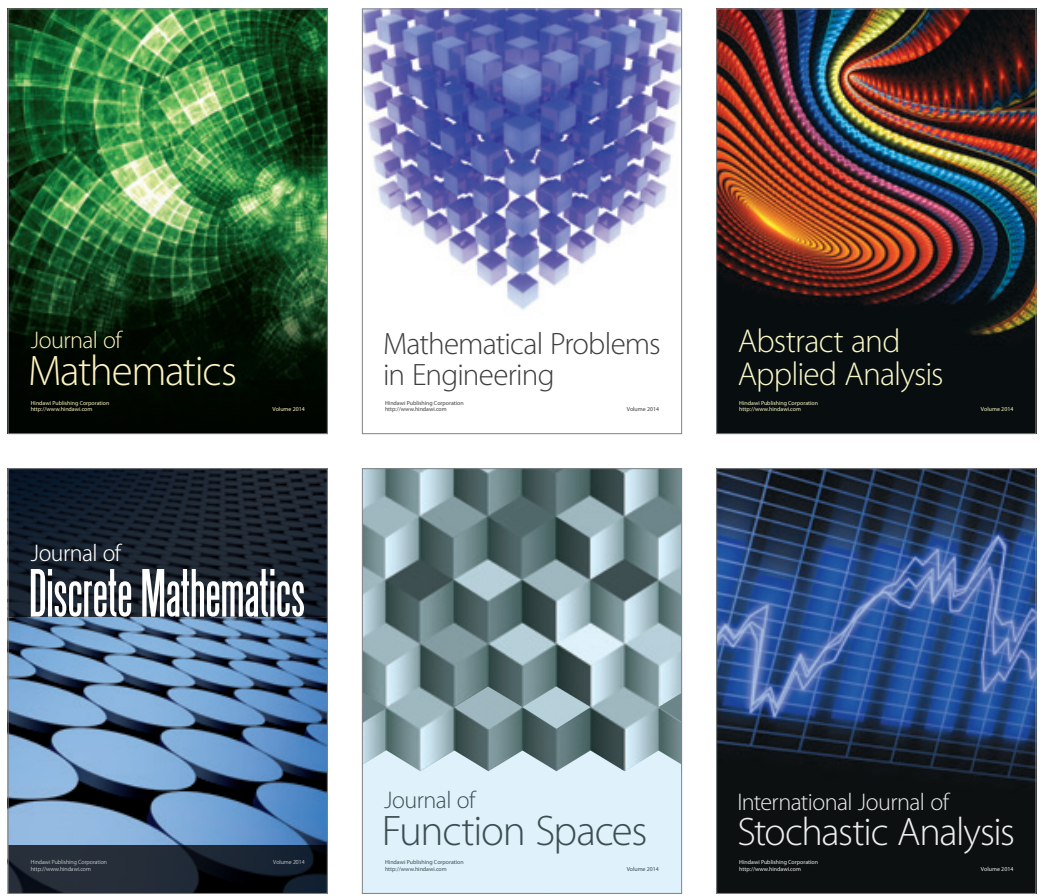

Journal of

Function Spaces

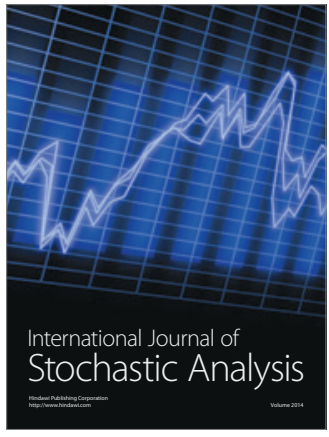

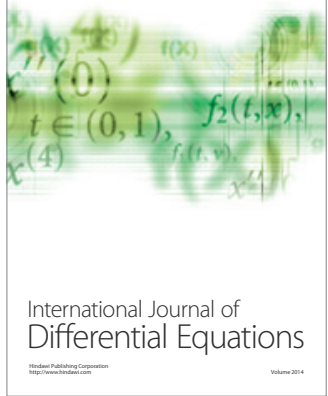
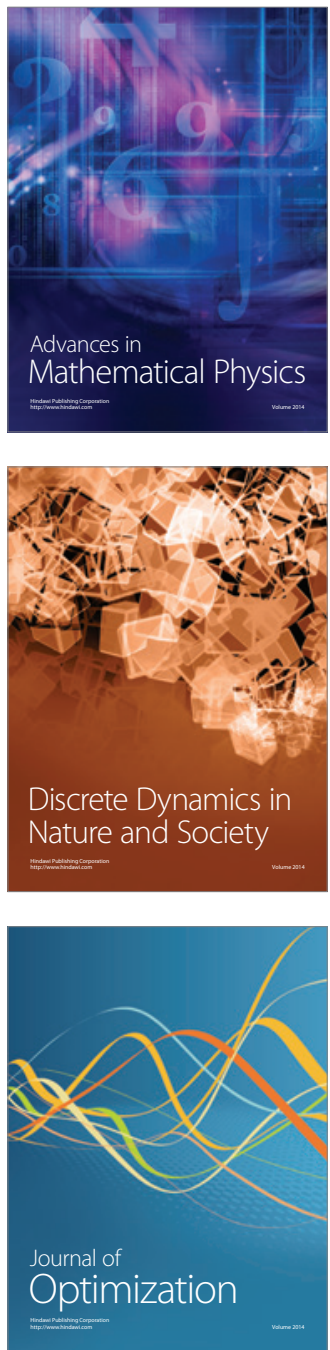